\title{
Power Controlling, Monitoring and Routing Center Enabled by a DC-Transformer ${ }^{\dagger}$
}

\author{
Syed Ashad Mustufa Younus ${ }^{1, *, \ddagger}$, Matteo Nardello ${ }^{2}$, Pietro Tosato ${ }^{2}$ and Davide Brunelli ${ }^{2}$ \\ 1 Department of Information Engineering and Computer Science, University of Trento, 38122 Trento, Italy \\ 2 Department of Industrial Engineering, University of Trento, 38122 Trento, Italy; \\ matteo.nardello@unitn.it (M.N.); pietro.tosato@unitn.it (P.T.); davide.brunelli@unitn.it (D.B.) \\ * Correspondence: syed.younus@unitn.it; Tel.: +353-899-763-739 \\ $+\quad$ This paper is an extended version of our paper published in IEEE-EESMS 2015, Title "Model of scalable \\ future consumer grid with residential power routing". \\ $\ddagger$ Current address: ICT Doctoral School, Via Sommarive, 9 I-38123 POVO, 38122 Trento, Italy.
}

Academic Editor: Chunhua Liu

Received: 1 February 2017; Accepted: 14 March 2017; Published: 21 March 2017

\begin{abstract}
The penetration of various types of renewable sources and on-site storage devices have recently focused attention towards DC power distribution in consumer grids to achieve the target of zero/positive energy buildings and communities. To achieve this target, the most important component is the DC consumer grid architecture which can integrate not only renewable sources and storage, but also enable the implementation in any conventional AC distribution network without any significant upgrade. To this end, a unique DC Transformer enabled DC microgrid architecture is presented in this paper. The architecture, called PCmRC (power controlling monitoring routing center) is proposed to manage distributed energy sources and storage at any stage and also directly interconnects the DC consumer grid with the conventional AC power grid. This paper also investigates detailed control algorithms of each component and the DC Transformer topology in addition to proposing four unique stages of grid operational modes to enhance the overall grid stability in any operational condition. The main objectives are to maximize the exploitation of renewable sources, to decrease reliance on fossil fuels, to boost the overall efficiency of the grid by reducing the power conversion losses and demand side management in all possible forms. The simulation platform is designed in MATLAB/Simulink. Simulation results of several types of case studies show the effectiveness of the proposed power distribution and management model.
\end{abstract}

Keywords: micro grids; sustainable energy; demand side management; DC Transformer (DCT); energy storage; consumer grid; power controlling monitoring and routing center (PCmRC)

\section{Introduction}

With the penetration of renewable energy sources, increasing energy demand and price of fossil fuels in society, many technologists have become confident that by 2030, the world's energy requirements will be provided mostly by renewable and distributed energy sources [1]. This is the reason why microgrids have become one of the hottest topics of renewable energy research and have been recognized as one of the 10 most emerging technologies in recent years [2]. As convectional fossil fuel based power generation plants are situated far from urban areas, the major challenges we are currently facing are associated with the transmission, distribution and storage of electricity. To reduce the transmission infrastructure cost and related losses, researchers have started studying microgrids [3]. Initially, research was mainly focused on AC microgrids due to the existing power generation architectures. More recently, due to the ease of exploitation of renewable energy sources, integration of economical battery storage solutions [4] and penetration of DC appliances in commercial, 
industrial and residential sectors, researchers have started investigating DC microgrids. DC microgrids boost efficiency by reducing the number of power converters, simplifying control, eliminating reactive power factor issues and delivering high power quality cost effectively.

Several studies have been done and DC microgrids implemented and tested in various applications such as naval ships, air-crafts [5], commercial data-centers [6,7], residential buildings [8], communities $[9,10]$ and smart cities [11]. Besides the above-mentioned advantages, DC microgrids bring several open issues and challenges, which need to be solved [12] such as (i) the integration of DC microgrids into the convectional AC distribution network [13]; (ii) availability of DC appliances [14]; (iii) how to manage the constant-current and inductive load within DC grids, and, most importantly, (iv) standard and scalable DC microgrid architecture and economical protection systems. To solve issues associated with the DC grids, a zonal microgrid concept is presented in [15], in which each independent consumer or building is considered as a zonal microgrid and connected to the AC main grid via a bidirectional AC/DC converter. Each zonal microgrid generates its own power and may take balance power from the main grid. Reference [16] proposed a centralized control with a high-speed communication link-a fast, efficient and economical method. However, the proposed micro-grid's reliability is dependent upon that link, as potential degradation of the communication link would cause reliability issues for the entire DC micro grid. To overcome such problems, many authors have proposed the distributed control of microgrids such as Droop control [17], DC-Bus Signaling [18], Modified DC bus (MDB) Signaling [19], active load sharing control [20,21], AC-signal modulation over DC voltage, circulating current method [22], and multi-agent based control [20,23]. However, the common issues are that the processing of each node, size and cost of the entire system would be increased drastically, continuous monitoring of SoC (state-of-charge) of battery-storage becomes complicated, overall grid control becomes slow and fault correction and isolation become complex [24].

To solve aforementioned issues, a DC Transformer (DCT) enabled consumer grid model named PCmRC (power controlling monitoring and routing center) is presented in this paper. The main motivation of the presented PCmRC consumer grid model is to make provisions for integration of any type of renewable or distributed sources and storage at any stage without any significant changes in the existing infrastructure. The proposed model can handle low/high voltage DC and AC powered load without any additional power converter. The PCmRC consists of a DCT, which provides magnetic isolation between the main distribution bus and the consumer load. It embeds over voltage and short circuit protection on each port: in case of emergencies, the switch will protect the grid, ensuring the overall grid stability and reliability.

\section{Description of DCT Enabled PCmRC Grid}

The envisioned DCT enabled consumer grid model is shown in Figure 1. The PCmRC is a scalable and standard model of a futuristic prosumer grid, which can be integrated into existing infrastructure without any significant modification. The proposed consumer grid architecture consists of one main DC voltage bus and sub DC buses. The main DC bus operates in the range between 350 to $400 \mathrm{~V}$ DC with nominal voltage of $380 \mathrm{VDC}$, used for electrical distribution and integration of high power local generators, storage and the AC main grid. This DC distribution voltage level is selected to comply with industrial DC grid standards such as the EMerge Alliance [25] and the European Telecommunication Standards Institute (ETSI). The ETSI (EN 300 132-3-1) standard allows up to 400 VDC for DC powered ICT equipment to be used in data centers. Moreover, IEC System Evaluation Group (SEG) 4 is also recommending DC voltage standards for low voltage DC (LVDC) distribution up to 1.5 KVDC [26]. The sub DC buses are used to power up the consumer side and drive both high power AC/DC loads up to $300 \mathrm{~V}$ and low voltage sensitive DC appliances up to $24 \mathrm{VDC}[25,26]$. The DCT is the key component and "Hub" of all activities including controlling bidirectional power flow, on-site low voltage storage management, grid protection, voltage regulation and fault isolation. Therefore, there is no need to install an additional expensive DC protection system, which helps to lower the overall operational grid cost. The motivation behind the standardized grid model is to reduce the production cost and fault 
rectification time. More details regarding LVDC consumer grid power management will be discussed in Section 3.

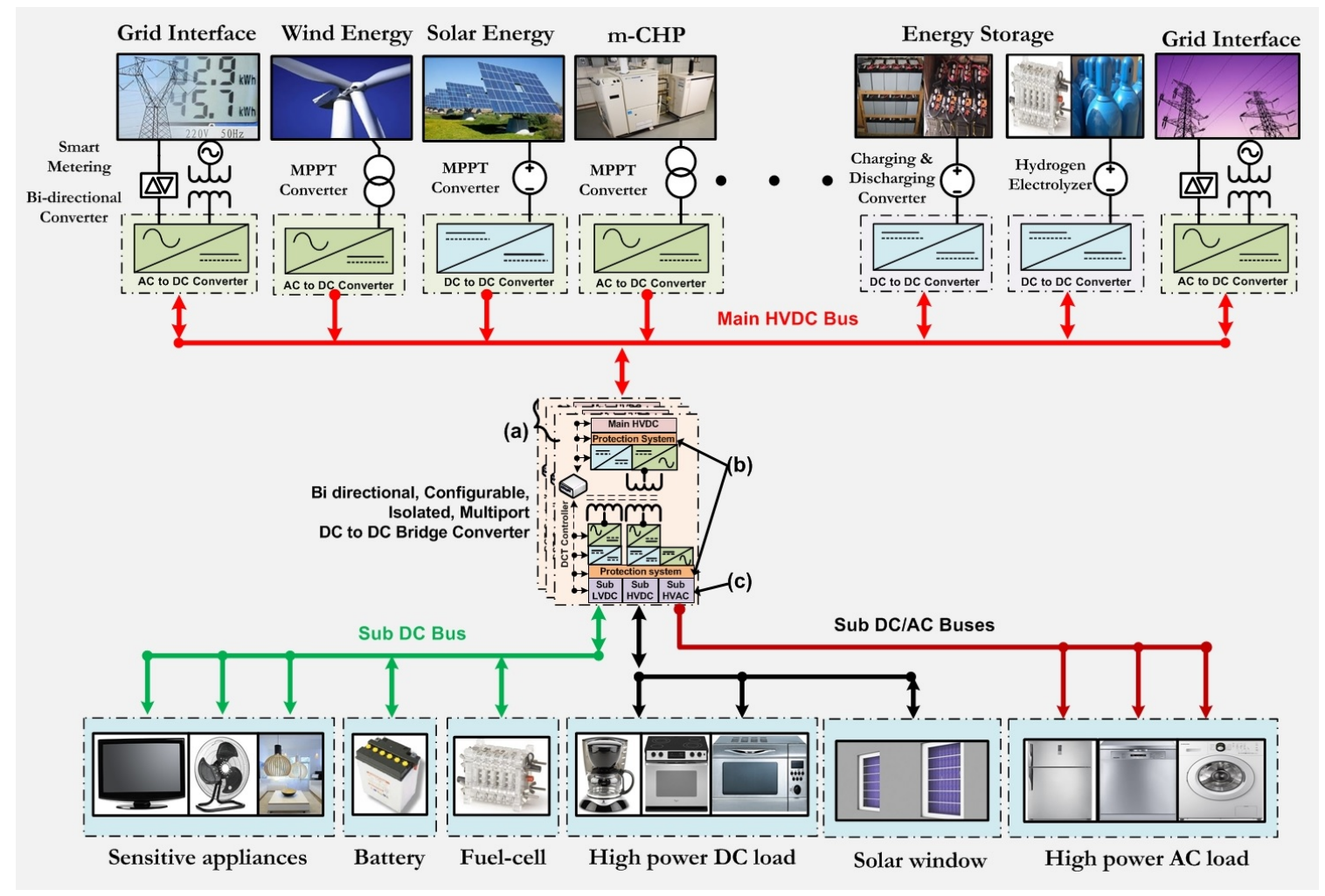

Figure 1. DCT enabled PCmRC consumer grid architecture. (a) multiple DCT module connected (b) built-in protection on each DCT terminal; and (c) dedicated terminal for AC load.

\subsection{DCT Topology}

The solid state transformer is one of the critical transmission devices used in flexible alternating current transmission systems (FACTs) [27]. The main motivation behind solid state transformers is to apply the state-of-the-art power electronic technology [13] to achieve high operating frequency in order to reduce the footprint of the transformer without affecting the power handling capacity. Figure 2 shows the triple-active-bridge (TAB) topology of the DCT under consideration. The reason for using TAB topology is to handle both AC/DC high voltage and low-voltage DC load at the same time without the need of additional converters. The DCT topology is comprised of the main DC bus interface and two sub DC bus interfaces. The main DC bus interface is directly connected to the main DC bus, which operates in the range between $350 \mathrm{~V}$ to $400 \mathrm{~V}$ DC and handles the bidirectional power flow between the main DC bus and sub DC buses in order to regulate the bus voltages. The sub high voltage DC interface is used to handle high voltage DC storage and non-critical DC and AC powered loads. The sub LVDC interface is used to handle the low voltage storage and critical sensitive electronic appliances. Thus, the DCT can be considered as a three port energy router and this characteristic makes it suitable to enable capability for handling both high and low voltage DC storage and AC loads with better performance as compared to conventional AC or DC micro grids. 


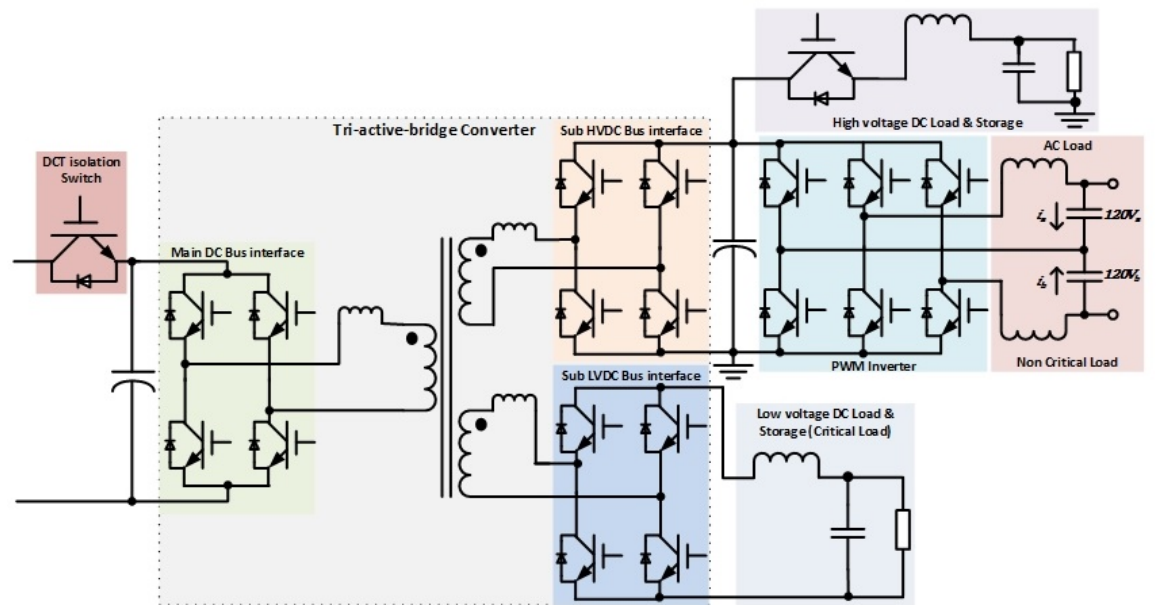

Figure 2. Triple-active-bridge topology used in DCT.

\subsection{Interfaces for Sustainable Sources and Storage}

Figure 3 shows the standard interface of renewable, distributed sources, storage and the bidirectional AC main grid for backup power. The standard interface makes sure that the output voltage of all sources connected to the common main DC bus would be at the same level. It also assures the reliability and scalability of the entire consumer grid. Every interface operates autonomously and there is no impact on grid performance and control algorithms due to increasing or decreasing the number of renewable sources, storage and AC main grid connections. The PCmRC consumer grid model always maximizes the exploitation of renewable sources and only takes balance power from the AC main grid to avoid load-shedding. Therefore, the PCmRC ensures the "plug-and-play" function of all types of micro-sources, storage and main grid connections. PCmRC only manages available power either from the main grid in the absence of renewable/distributed energy sources or from renewable sources in the off-grid/islanding condition without any upgrade.

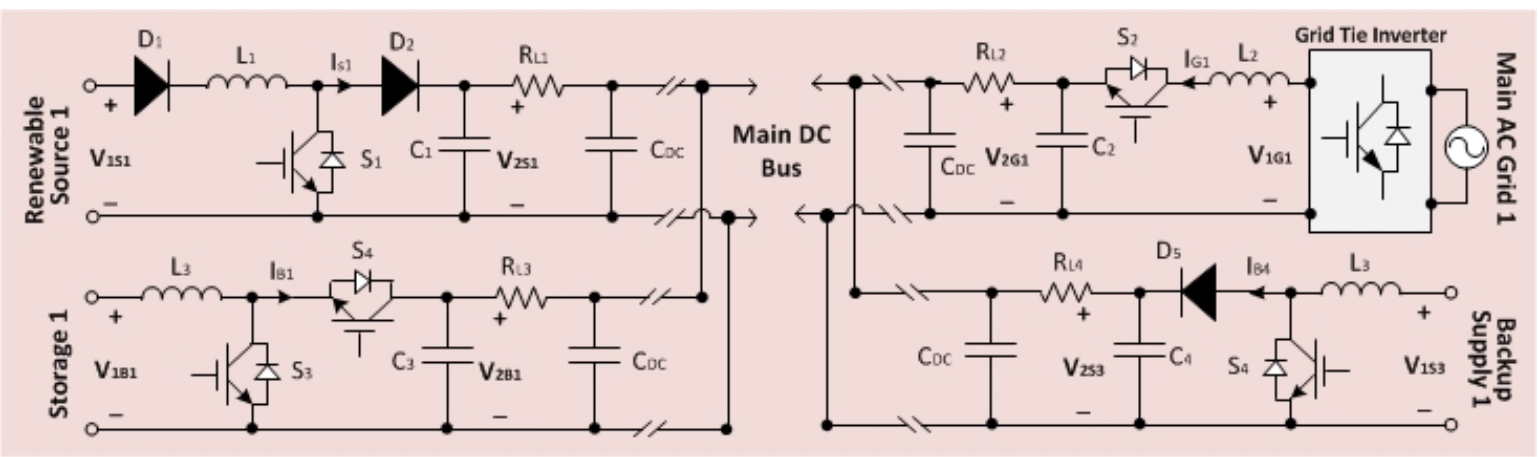

Figure 3. Standard interface for power sources and storage.

\subsection{Load Classification and Demand Side Management}

The main function of the local generators and storage system is to ensure the reliable and continuous supply of power to loads by reducing the dependency on the AC main grid. Therefore, demand management is the key category for microgrid control. The unique power distribution scheme used in PCmRC discriminates the priority level of the load. Of course, it can handle both AC and DC power loads connected to the consumer grid. The priority level is defined considering the consumer requirements and can fall into two categories, i.e., critical and noncritical loads. Taking as an example the worst case scenario, during a main grid fault, in which the energy coming from renewable sources and storage is not enough to fulfill all power requirements, the PCmRC is able to switch off noncritical 
load and to supply power only to the critical loads. In this way, PCmRC allows consumers to configure the priority level of the loads depending upon the importance and utilize the energy storage in more efficient way.

\subsection{Protection and Fault Isolation}

The DCT module is used for power distribution and demand side load management. Each DCT continuously monitors both main bus and sub DC bus interfaces. On detecting any anomalous situation such as over/under voltage and short-circuit current, the respected DCT(s) immediately switch(es)-off and isolate(s) the fault within the grid. Moreover, battery storage can be directly coupled on the sub LVDC bus ( $24 \mathrm{~V}$ ) without any additional converters. Therefore, it has voltage-sag ride through capability, which increases the overall reliability and protection for sensitive electronic loads.

\section{LVDC Consumer Grid Power Management}

Zero or positive energy buildings and communities are a promising and realistic concept. According to Article 9 of the Energy Performance Buildings directive (EPBD), member states shall ensure that, by the end of 2020 (2018 for public buildings), all new buildings are nearly zero-energy [28]. The full exploitation of renewable sources and efficient integration of storage devices with an effective and reliable consumer grid control model is required to realize zero/positive energy buildings and communities. Each house or building is considered as a microgrid; in this way, each micro grid is responsible for generating its own power by using distributed and sustainable energy sources. The PCmRC consumer grid model provides a standard interface for all types of renewable sources, storage, local generators and AC main grid interfaces, as shown in Figure 3. The DCT module is connected between the main DC distribution bus, and sub DC buses are connected on the consumer side. There are two main purposes of the main DC bus, firstly to integrate all local DCT modules into the same bus and secondly to integrate the large scale generators, backup-supplies and AC main grid connections. In this way, all local zonal DC micro grids can share power locally because the load of each consumer grid is not constant, and in the case of any distributed zonal grid being unable to utilize the storage locally, it can then share the surplus power with other adjacent microgrids. The more important factor in the DC microgrid is that there is solely one control parameter (i.e., DC bus voltage) to consider [24]. Therefore, in case the bus voltage starts to drop, each zonal microgrid starts pumping surplus power from locally generated sources and storage to the common main DC bus, regulating the DC bus voltage up to an optimum level. However, in this paper, only one consumer grid model is discussed and this concept (model) will be the same for the other zonal consumer grids.

In order to operate the DCT module as an intelligent energy router, the DCT module must have the ability of operating in different conditions. Most researchers propose three stages of grid operational modes as discussed in $[15,24,29]$. However, in the PCmRC consumer grid model, we propose four stages (excluding the buffer stage), in order to get effective control on power flow and increase the overall grid reliability. The different operational modes flow diagram is shown in Figure 4.

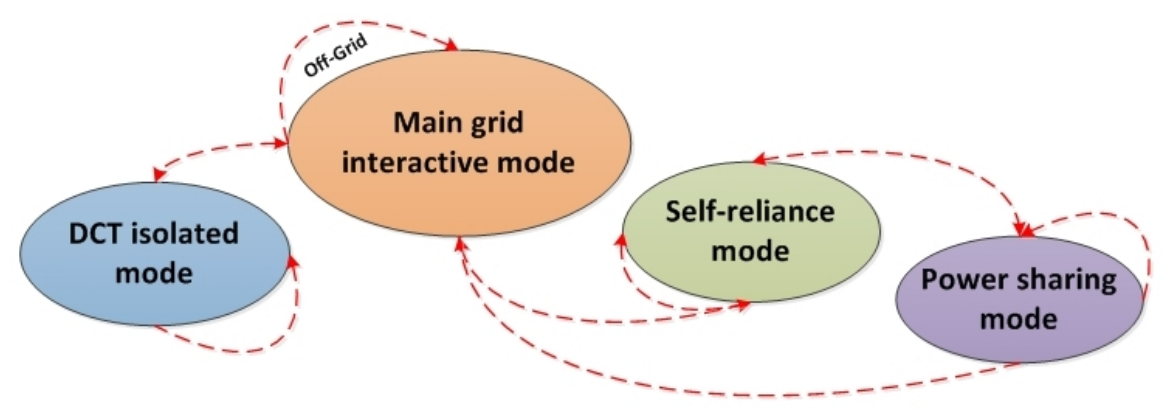

Figure 4. PCmRC operational modes flow diagram. 
In DCT isolated mode, the power required by the load is greater than the locally available power sources and the whole grid is operating in an islanding or off-grid condition, as mentioned in Equation (1). Therefore, the main bus voltage is starting to decrease until it reaches point $\mathrm{A}$, as shown in Figure 5. Then, each connected DCT starts working in isolated mode and there will be no power flow from sub DC bus to main DC bus. In this mode, each DCT module switches off the non-critical AC and DC loads so that the sub HVDC bus voltage becomes "zero volt", if there is no storage connected to the sub HVDC bus. The locally available storage is solely utilized to operate only critical loads connected to the sub LVDC bus, until the storage depletes or the back-supply/grid interface starts delivering power to the microgrid to avoid load-shedding. The detail voltage levels are mentioned in Table 1 and the variable abbreviations are given in Table 2:

$$
\begin{gathered}
\left(P_{S L O} \geq\left(P_{M S I}+P_{H S I}+P_{L S I}\right)\right. \\
\because P_{R S I}+P_{D G I}+P_{M G I}=0\left(P_{H L O}=0\right) .
\end{gathered}
$$

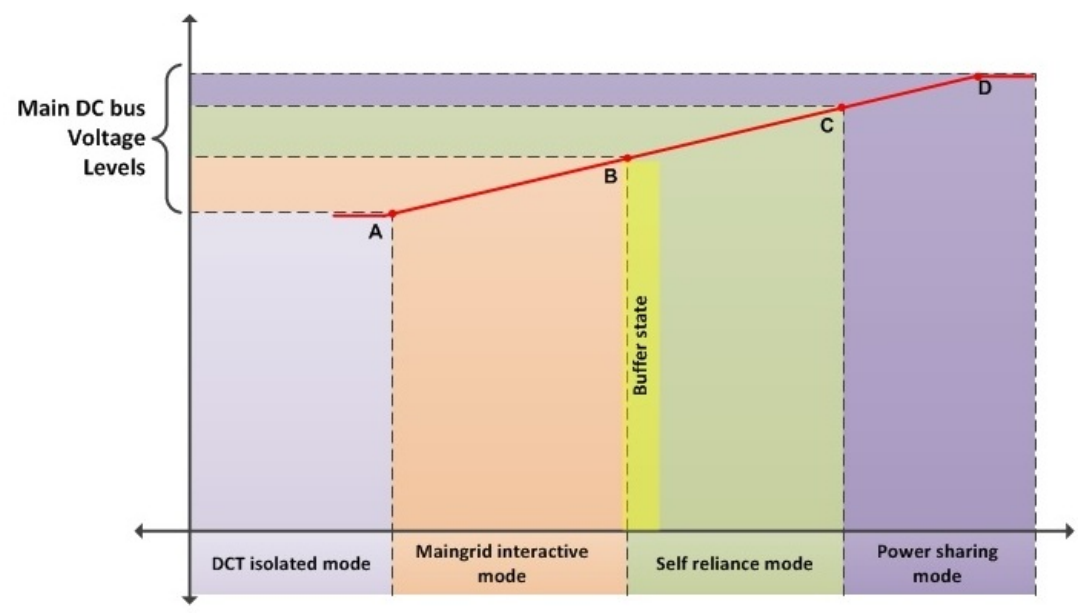

Figure 5. PCmRC operational modes graphical diagram.

Table 1. List of key parameters of the PCmRC Model.

\begin{tabular}{ccccc}
\hline $\begin{array}{c}\text { Mode of } \\
\text { Operation }\end{array}$ & Main DC Bus & $\begin{array}{c}\text { Sub HVDC } \\
\text { Bus }\end{array}$ & $\begin{array}{c}\text { Sub LVDC } \\
\text { Bus }\end{array}$ & Power Flow \\
\hline $\begin{array}{c}\text { DCT } \\
\text { Isolated }\end{array}$ & $\begin{array}{c}V_{M B} \leq \\
355 \mathrm{VDC}\end{array}$ & $V_{S H B}=0$ & $V_{S L B} \leq 24.2 \mathrm{~V}$ & $\begin{array}{c}\text { Power coming from locally available } \\
\text { storage only. High power AC /DC load is } \\
\text { switched off }\end{array}$ \\
\hline $\begin{array}{c}\text { Main Grid } \\
\text { Interactive }\end{array}$ & $\begin{array}{c}360 \mathrm{~V} \leq V_{M B} \\
\leq 375 \mathrm{~V}\end{array}$ & $V_{S H B} \geq 280 \mathrm{~V}$ & $\begin{array}{c}24.8 \mathrm{~V} \leq V_{S L B} \\
\leq 25.9 \mathrm{~V}\end{array}$ & $\begin{array}{c}\text { Power coming from renewable sources and } \\
\text { AC main grid. All types of storage are } \\
\text { kept isolated }\end{array}$ \\
\hline $\begin{array}{c}\text { Buffer } \\
\text { State }\end{array}$ & $\begin{array}{c}376 \mathrm{~V} \leq V_{M B} \\
\leq 380 \mathrm{~V}\end{array}$ & $\begin{array}{c}280 \mathrm{~V} \leq V_{S H B} \\
\leq 300 \mathrm{~V}\end{array}$ & $\begin{array}{c}26 \mathrm{~V} \leq V_{S L B} \\
\leq 28.1 \mathrm{~V}\end{array}$ & $\begin{array}{c}\text { Power coming from renewable source and } \\
\text { main grid. All types of storage are } \\
\text { connected as a Load }\end{array}$ \\
\hline $\begin{array}{c}\text { Self-Reliance } \\
\begin{array}{c}381 \mathrm{~V} \leq V_{M B} \\
\leq 395 \mathrm{~V}\end{array}\end{array}$ & $\begin{array}{c}280 \mathrm{~V} \leq V_{S H B} \\
\leq 300 \mathrm{~V}\end{array}$ & $\begin{array}{c}28.2 \mathrm{~V} \leq V_{S L B} \\
\leq 28.8 \mathrm{~V}\end{array}$ & $\begin{array}{c}\text { Power coming from renewable sources only. } \\
\text { Maingrid is isolated. All types of storage } \\
\text { are connected as a load }\end{array}$ \\
\hline $\begin{array}{c}\text { Power } \\
\text { Sharing }\end{array}$ & $V_{M B} \gg 395 \mathrm{~V}$ & $V_{S H B} \gg 300 \mathrm{~V}$ & $\begin{array}{c}28.8 \mathrm{~V} \leq V_{S L B} \\
\leq 29.5 \mathrm{~V}\end{array}$ & $\begin{array}{c}\text { Power coming from renewable sources only. } \\
\text { Maingrid and all type of storage are } \\
\text { connected as a load }\end{array}$ \\
\hline
\end{tabular}


Table 2. Nomenclature.

\begin{tabular}{|c|c|}
\hline$P_{H L O}$ & The High Power AC and DC Load output \\
\hline$P_{S L O}$ & The Low voltage sensitive electronics load Output \\
\hline$P_{R S I}$ & The Input Power from Renewable sources \\
\hline$P_{D G I}$ & The Input Power from local distributed generators \\
\hline$P_{M S I}$ & The Input Power from storage integrated with Main DC bus \\
\hline$P_{H S I}$ & The Input Power from storage integrated with Sub HVDC bus \\
\hline$P_{L S I}$ & The Input Power from storage integrated with Sub LVDC bus \\
\hline$P_{M G I}$ & The input Power from AC main grid \\
\hline$V_{M B}$ & The Main DC bus voltage \\
\hline$V_{S H B}$ & The Sub HVDC bus voltage \\
\hline$V_{S L B}$ & The Sub LVDC bus voltage \\
\hline$v_{m}$ & Secondary DC link voltage \\
\hline$v_{m r e f}$ & Secondary DC link voltage reference \\
\hline$i_{S}$ & Input primary current \\
\hline$i_{s d}$ & $d$-axis current \\
\hline$i_{s q}$ & $q$-axis current \\
\hline$i_{\text {sdref }}$ & $d$-axis current reference \\
\hline$i_{\text {sqref }}$ & $q$-axis current reference \\
\hline$v_{S}$ & Input primary voltage \\
\hline$v_{s d}$ & $d$-axis voltage of $v_{s}$ \\
\hline$v_{s q}$ & $q$-axis voltage of $v_{s}$ \\
\hline$V_{l}$ & Output side low DC bus voltage \\
\hline$V_{\text {lref }}$ & Output side low DC bus voltage reference \\
\hline$\varphi$ & Phase shift \\
\hline$V_{h}$ & Input side high DC link voltage \\
\hline$f$ & Switching frequency \\
\hline$L$ & Leakage inductance \\
\hline So $C_{\text {bat }}$ & State of Charge of battery \\
\hline$i_{b a t}$ & Battery storage current connected to sub DC bus \\
\hline$i_{\text {batref }}$ & Battery storage current reference \\
\hline
\end{tabular}

In the main grid interactive mode, the output power (connected load) of the grid is greater than the generated power from renewable sources and local distributed generators. However, the main grid interface and storage is available. In this case, PCmRC will only take the balance power from the main grid to fulfill the load requirements and keep the sub DC buses within certain voltage limits as shown in Table 1 and in Equation (2). If the sub voltage buses operate in these operational limits, then PCmRC will keep the storage isolated. In the main grid interactive mode, PCmRC operates the grid in the region between points A and B, as shown in Figure 5. In this region, only balance power is taken from the main grid interface just to power up the load connected to the sub HVDC and LVDC buses and storage is kept isolated and considered to take "zero current", as shown in Equation (2):

$$
\begin{gathered}
\left(P_{H L O}+P_{S L O}\right)=\left(P_{R S I}+P_{D G I}+P_{M G I}\right) \\
\because P_{M S I}+P_{H S I}+P_{L S I}=0 .
\end{gathered}
$$

If the output power or load connected to the grid is less than or equal to the power generated by the renewable sources and consumer-grid can fulfill demand side power requirements without taking power from the AC main grid, the PCmRC is working in self-reliance mode.

In this mode, PCmRC ensures the power supply to both critical and non-critical loads and balance power will be used to charge the storage connected to DC buses. In Figure 5, the region between points $\mathrm{B}$ and $\mathrm{C}$ is the self-reliance region and in this mode the energy storage is treated as a load, as shown in Equation (3):

$$
\begin{gathered}
\left(P_{H L O}+P_{S L O}\right) \leq\left(P_{R S I}+P_{D G I}-P_{M S I}-\right. \\
\left.P_{H S I}-P_{L S I}\right) \because P_{M G I}=0 .
\end{gathered}
$$


In case of storage fully charged and surplus energy generated by local generators, the PCmRC will start sharing surplus power to the AC main grid, which is called power sharing mode. In this mode, the main grid is also treated as a load, and balance power is sent to the AC main grid via a grid-tie inverter, as shown in Figure 3 and Equation (4):

$$
\left(P_{H L O}+P_{S L O}\right) \leq\left(P_{R S I}+P_{D G I}-P_{M S I}-P_{H S I}-P_{L S I}-P_{M G I}\right) .
$$

Renewable sources of energy are not constant in time, and, due to their nature, they are not suitable to fulfill the requirements of the variable loads connected to the grid. The stochastic behavior of distributed energy resources can cause problems to the stability of the grid. To avoid such scenarios, PCmRC introduces a buffer stage while moving from the main grid interactive mode to the self-reliance mode in order to meet the unpredicted load requirements and to increase the grid reliability. Another reason to use buffer stage is that, in the main grid interactive mode, the AC main grid is used to supply power solely to connected loads and storage is kept isolated. As renewable sources' output power increases gradually [10], as soon it becomes equal to the load power requirement, the PCmRC enters into self-reliance mode at point B, shown in Figure 5. However, due to bad weather conditions or sudden increase in demand side power requirements, the power generated from renewable sources might not be enough to meet the unpredicted change, and, at that stage, the available storage is also not charged enough to compensate for the deficiency of power. Therefore, the buffer stage is introduced, in which PCmRC starts charging the storage from surplus energy coming from renewable sources and makes sure the level of SoC of storage will be enough before transition between the main grid interactive mode to the self-reliance mode without compromising grid reliability. In Figure 5, the highlighted area is the buffer stage and the bus voltages are mentioned in Table 1.

\section{Overall Grid Control Design}

The conventional AC microgrid usually operates in two modes such as grid connected mode and islanded mode [30], and it has different control and operation methods for both scenarios. However, in the DC consumer grid, the mode of operation is slightly different; for example, the utility grid is connected through the AC-DC bidirectional converter, and, depending on the bus voltage, the interface converter allows for exchanging power either with a utility grid to consumer grid or vice versa. To demonstrate, the DCT enabled PCmRC power management strategy and detailed control scheme is outlined in this section. The main DC bus must be well maintained and operates between the rigid upper and lower limits. In case of any anomalous condition, the DC bus voltage can collapse the whole DC microgrid operation. Therefore, accurate meters such as [31] and a robust and reliable control and management scheme are required for the effective and sustainable consumer grid operation. The overall flow chart of the PCmRC consumer model is shown in Figure 6, which is fully compatible for off-grid and conventional grid connected operations. The PCmRC consumer grid consists of the following terminals. 


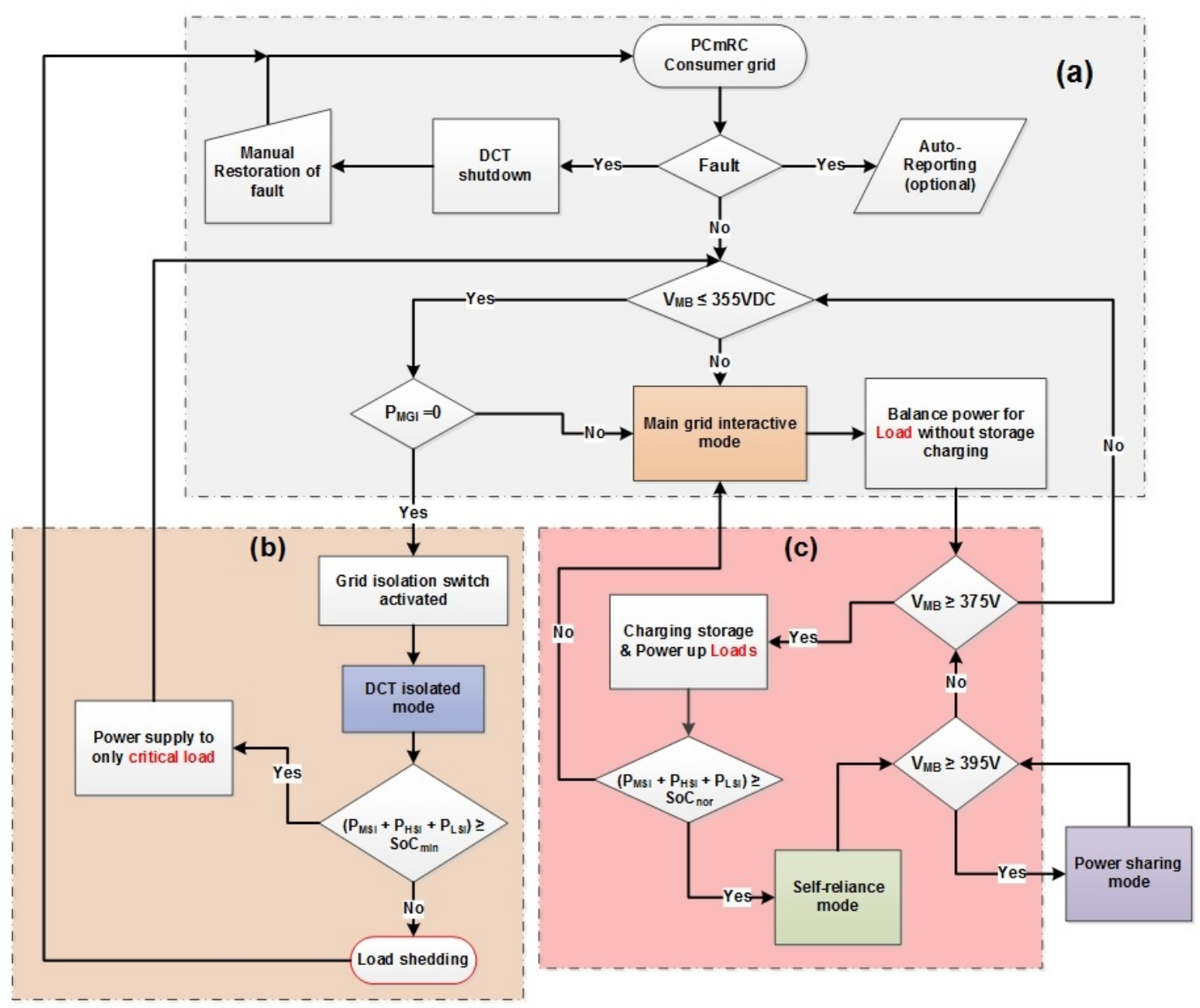

Figure 6. The overall operational control flow chart of PCmRC consumer grid model (a) in the case of the grid connected mode; (b) in the case of the DCT isolated mode; (c) in the case of the off-grid mode.

\subsection{DCT Control}

The DCT is the brain and hub of all power management in the PCmRC model. It provides $24 \mathrm{~V}$ on sub LVDC bus, $300 \mathrm{~V}$ on sub HVDC bus for high voltage DC and single phase AC output. The three-stage DCT control diagram is presented in Figure 7 and symbols used in the control diagram are elaborated in Table 2. The DCT converts AC to AC for step-up or step-down voltage as the conventional electrical transformer does, but in a more efficient way with embedded protection and a small footprint. As shown in Figure 2, the DCT consists of three I/O ports with high frequency transformers: DC to DC, AC to DC and DC to AC conversions. In normal operation, the power is transferred from the main voltage bus to the sub voltage buses and the sub LVDC/HVDC H-bridge works as a control rectifier. The $\mathrm{d}$-q vector control diagram of a single-phase rectifier is shown in Figure 7a. Both a high voltage DC main bus and the power factor are controlled by a dual loop controller and manage the real and imaginary values of the current and voltage. The outer and inner loop of the $d$-axis component is controlling the active power control loop (both voltage regulator and current loop). The $q$-axis is controlling the reactive power control loop, and its reference is set to zero $\left(i_{\text {sqref }}=0\right)$ for unity power factor operation [27]. The triple-active bridge topology used in the DCT offers zero voltage switching, less stress on switches and bidirectional power flow. The difference in the phase shift determines the power transfer $\left(P_{\text {Output }}\right)$ between the main DC bus to sub DC buses [27]. Therefore, phase shift $(\varphi)$ control is used in the DC to DC conversion stage as shown in Figure $7 \mathrm{~b}$. The power transfer is controlled by configuring the phase shift on both the primary H-bridge and 
the secondary H-bridge side $\left(V_{h}\right)$, which regulates the sub DC voltage bus to the appropriate voltage levels as given by following Equation (5):

$$
P_{\text {Output }}=\left(V_{h}\right) / 2 L f \varphi(1-\varphi) .
$$

The inverter stage uses the capacitor current feedback control for a better voltage output by sensing the current from output capacitor. The inner loop is controlled by the capacitor current, and output AC voltage is controlled by the convectional PI controller as the outer loop, as shown in Figure 7c. A split phase inverter topology is used in the DCT, which supports $120 \mathrm{~V}$ and $240 \mathrm{~V}$ AC voltages regulated outputs as shown in Figure 2.

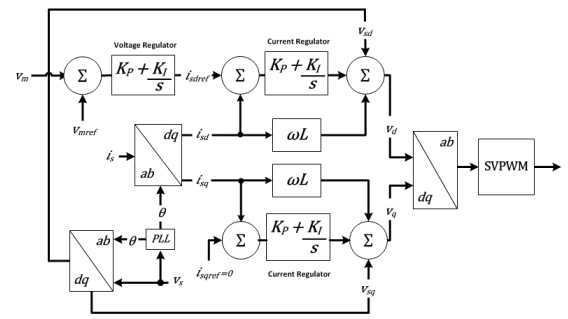

(a)

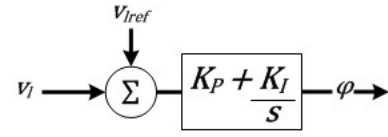

(b)

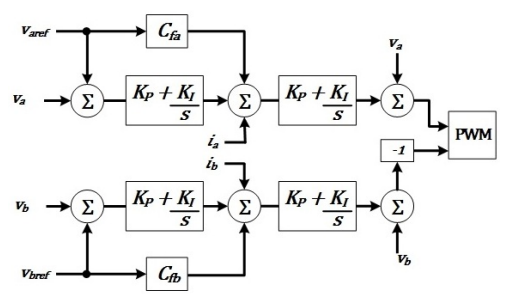

(c)

Figure 7. Control diagram of proposed DCT (a) high voltage AC to DC control stage; (b) DC to DC control stage; and (c) sub voltage DC to AC inverter control stage.

\subsection{Battery Storage Control}

The DCT enabled PCmRC architecture can accommodate both distributed and local energy storage. The distributed energy storage is connected to the main DC bus, and low voltage battery storage is connected to the sub DC buses. However, the distributed energy storage is considered as a backup source and it operates autonomously. The interface diagram of the energy storage is shown in Figure 3, which allows the bidirectional flow of the current depending upon the State of Charge (SoC) of the battery and operational mode of the PCmRC grid. The operation of the battery storage connected to the sub DC buses is dependent upon the State of Charge $\left(\mathrm{SoC}_{b a t}\right)$ of the battery. The PCmRC allows for consuming the available storage as long as $\mathrm{SoC}_{b a t}$ is within limits. Therefore, PCmRC operates the locally connected battery storage in three modes: charging, discharging and standby. The PCmRC always charges the battery storage only in self-reliance or power sharing modes and standby in main grid interactive mode. It exploits the locally available storage in the DCT isolated mode to power up the critical loads only. The PCmRC only charges the locally available storage from surplus power generated by the renewable sources and never charges the storage from the main grid supply to reduce the dependency on AC main grid. It also helps to reduce the carbon emissions. The detail control flow diagram including battery management is shown in Figure 6. In the worst condition, when the renewable sources are not available and the PCmRC consumer grid is operating in islanding condition, locally connected storage regulates the sub LVDC bus and the system operates in the DCT isolated mode. As shown in Figure 8, the outer voltage loop is cascaded with the battery current inner loop, until the $\mathrm{SoC}_{b a t}$ reaches down and equals the $\mathrm{SoC}_{\min }$ to avoid over discharging of the battery storage.

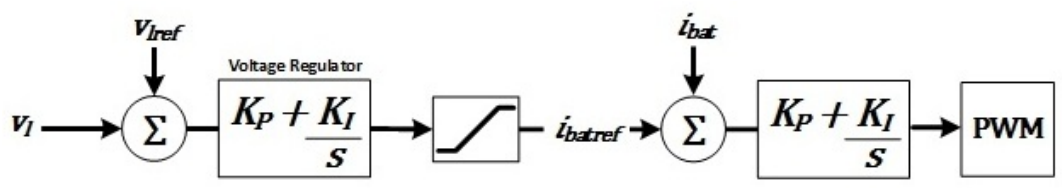

Figure 8. Battery SoC control diagram. 


\subsection{Grid Connected, Islanding and Off-Grid Control}

PCmRC considers the AC main grid connection as a finite energy source and the main grid is always connected through the standard interface to the main DC bus like any other sources, as shown in Figure 3. Therefore, multiple grid connections can be interfaced to the PCmRC in order to increase the redundancy, reliability and power sharing requirements with the adjacent grids. Moreover, the PCmRC control algorithm would be the same for grid-connected, islanding and off-grid operations without any upgrade. In case of convectional power distribution where the main grid is the only source of power and there is no local generator available, the PCmRC can manage the AC main grid supply to power up the loads, as shown in Figure 6a. Therefore, PCmRC architecture is perfectly compatible with existing infrastructure. If there is no source available locally and the consumer grid is operating in islanding mode, the PCmRC will provide power only to critical loads, until the storage depletes, as shown in Figure 6b. In case there is no grid-connection or consumer grid installed at a remote location, then PCmRC will operate the whole consumer grid on locally available sources and storage. However, the local generators must supply enough power to fulfill load requirements, as shown in Figure 6c.

\section{PCmRC System Level Case Study}

In order to verify the proposed PCmRC consumer grid model and power management strategy, a simulation model of each control module is built in the Matlab/Simulink (Mathlab 2016b) based on the architecture mentioned in Figure 1. The average model technique is used instead of the switching model in order to cover large scale power system dynamics. Moreover, the average modeling technique speeds up the simulation without losing key characteristics of the system. In the simulations, the power rating of the DCT module is set under $5 \mathrm{kVA}$ to simulate a typical residential consumer grid. Other important parameters are: LVDC load is $750 \mathrm{~W}$, LVDC storage is $550 \mathrm{~W}$, AC load is $2.5 \mathrm{kVA}, \mathrm{AC}$ main grid output voltage is $357 \mathrm{~V}$ and the HVDC bus voltage is $305 \mathrm{~V}$. Different scenarios of sources and loads profiles are simulated; however, due to page constraints, only key waveforms are shown and explained in this section.

\subsection{Grid Connected Mode without Renewable Sources and Storage}

When the entire consumer grid operates solely on the power coming from the main grid, PCmRC operates in the main grid interactive mode; there are different possibilities to enter in Grid connected mode, few of them are discussed in this section. As soon as the DCT detects that the main DC bus voltage $\left(V_{M B}\right)$ is less than $380 \mathrm{~V}$ and $V_{M B}$ is decreasing down to $375 \mathrm{~V}$, then, in order to avoid load-shedding, PCmRC enters into the main grid interactive mode. In this mode, PCmRC regulates the sub DC voltage buses and powers up the critical and non-critical loads as described previously (Figure 9a). At time $t=1.5 \mathrm{~s}$, when the $V_{M B}$ crosses the $375 \mathrm{~V}$ threshold, the main grid interface converter is then switched on and PCmRC manages the main grid power to regulate the main DC bus voltage in between $360 \mathrm{~V} \leq V_{M B} \leq 375 \mathrm{~V}$.

Before entering into the main grid interactive mode, DCT regulates the sub LVDC bus voltage to $\leq 26 \mathrm{~V}$, in order to avoid low voltage storage charging. In Figure 9b, the storage was charged with a constant current of $20 \mathrm{~A}$. As soon as $V_{M B}$ decreases from $377 \mathrm{~V}$, DCT then regulates the sub LVDC bus to less than $26 \mathrm{~V}$ because the control algorithm of PCmRC is designed in such a way that no storage would be charged on the low voltage side, if the sub LVDC voltage is less than $26 \mathrm{~V}$. Therefore, at $t=1 \mathrm{~s}$, the PCmRC switches off storage charging and only powers up the loads, as shown in Figure $9 b, c$. 


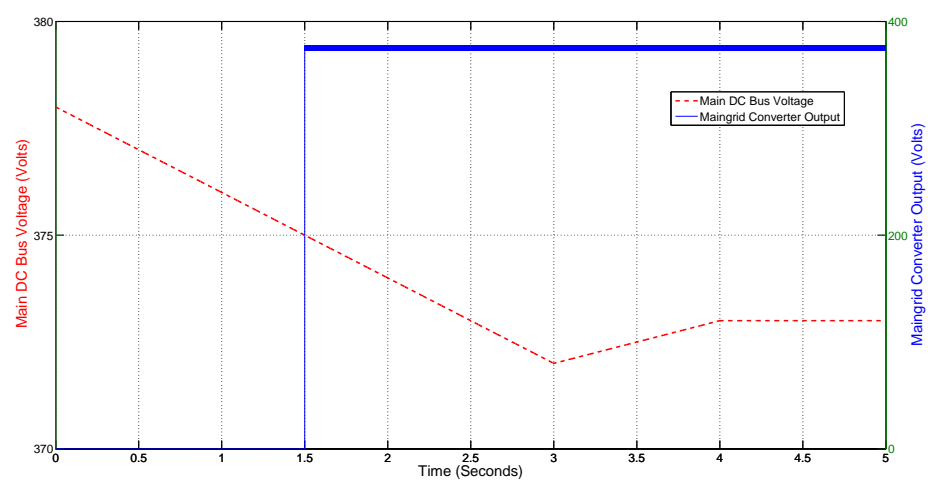

(a)

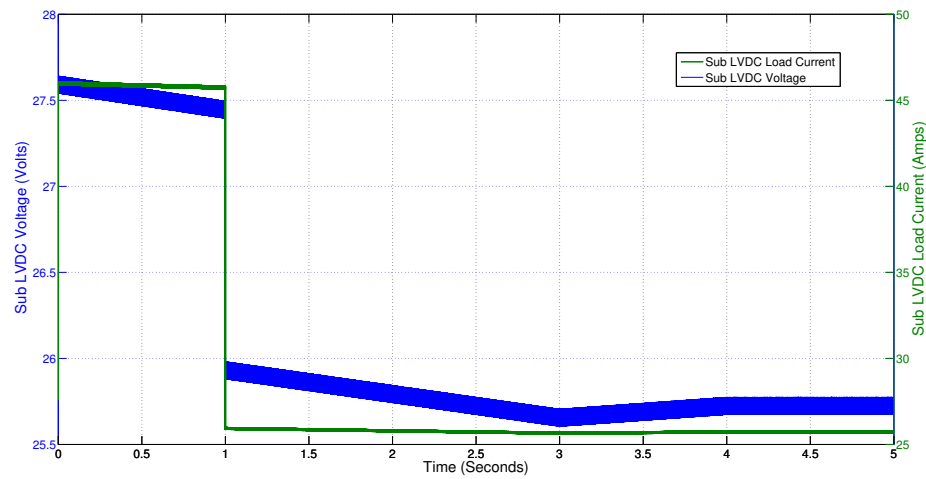

(b)

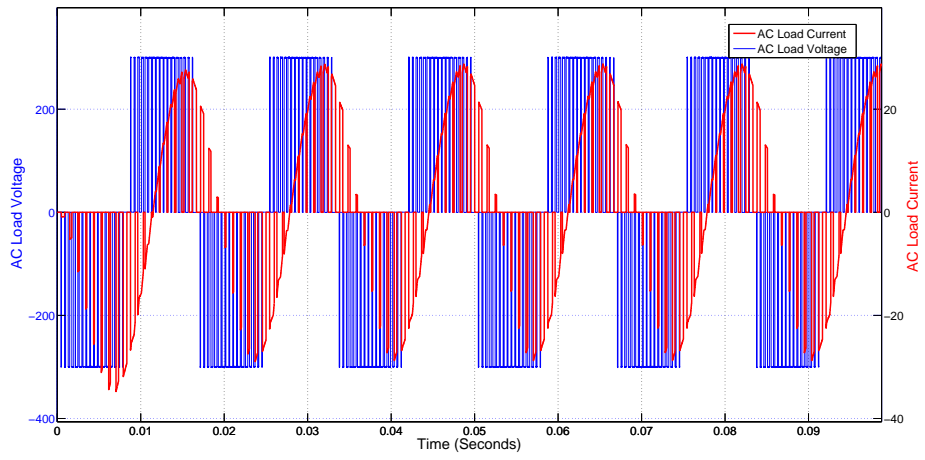

(c)

Figure 9. Simulation waveform during grid-connected operational mode (a) main DC bus and main grid converter output voltage waveform; (b) sub DC voltage and load current waveform; and (c) AC load voltage and current waveform.

\subsection{Peak Surplus Energy Generation and On-Site Storage}

If the locally available sources are generating surplus power that is more than the consumer demand, the PCmRC will operate the local consumer grid using solely the on-site available sources, operating in the so-called self-reliance mode. In Figure 10b, the slope of the $V_{M B}$ is positive and even though sufficient surplus power is coming from the on-site generators, in order to increase the grid reliability and stability, PCmRC grid operates in buffer state and switches off the main grid converter after $V_{M B}$ reaches $380 \mathrm{~V}$. However, in the buffer state, PCmRC starts charging the storage (at time $t=2 \mathrm{~s}$ in Figure 10a from the surplus energy to avoid any abnormal situations and ensures continuous supply for critical loads, as shown in Figure 10c. During the entire operation, stable power outputs are available for non-critical loads, as shown in Figure 10c. 


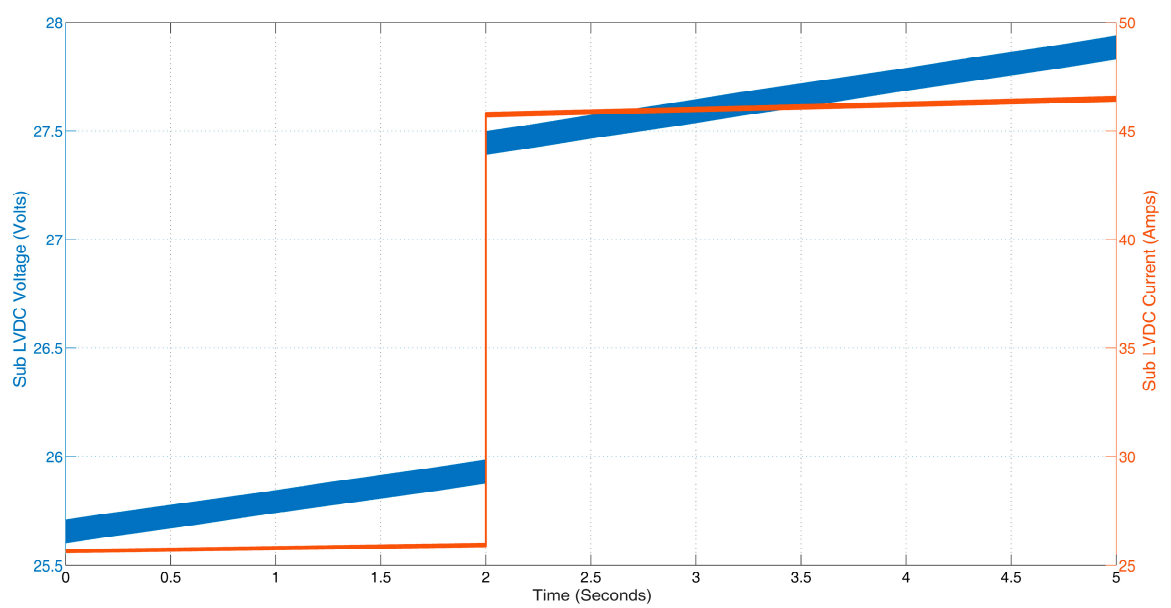

(a)

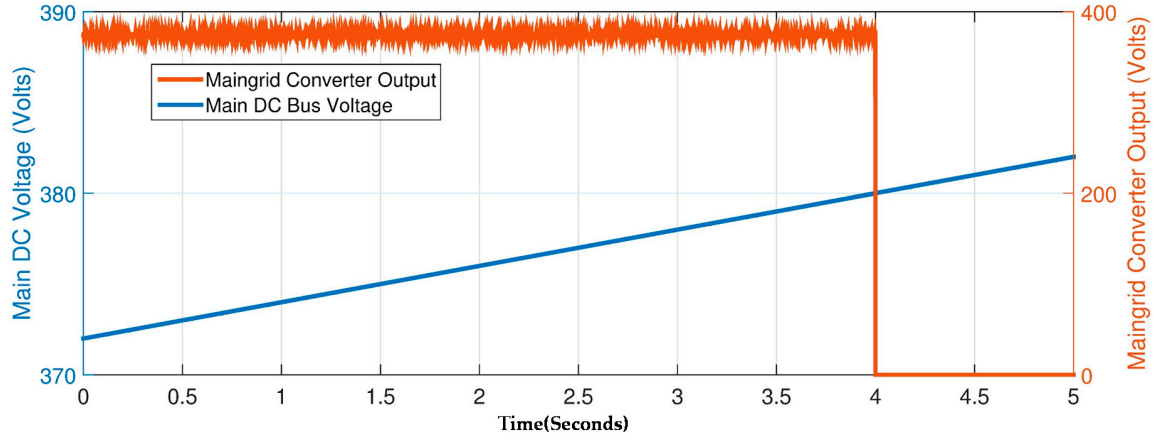

(b)

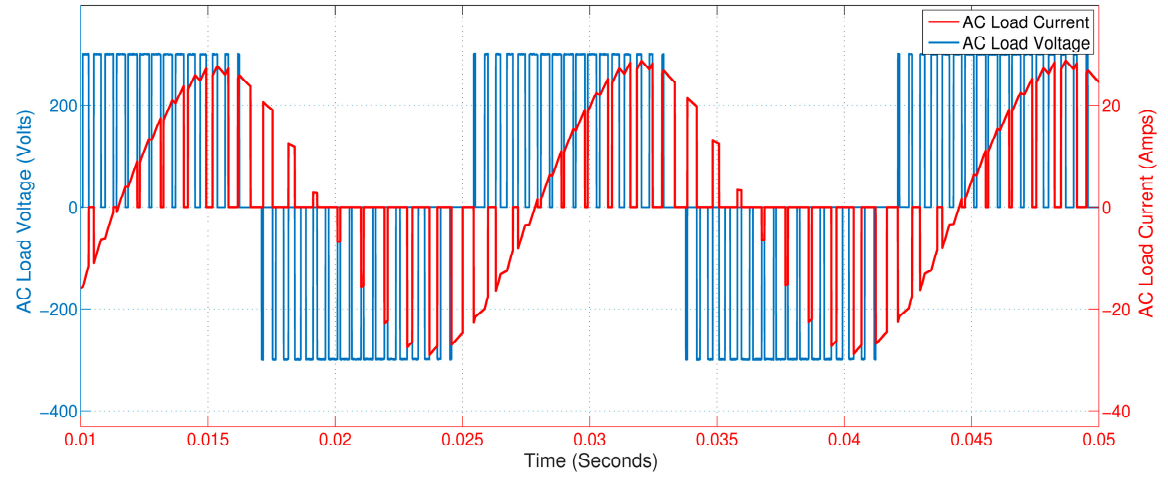

(c)

Figure 10. Simulated waveform during self reliance mode (a) sub LVDC voltage and current; (b) main $\mathrm{DC}$ bus and AC main grid converter output voltage; and (c) AC load voltage and current.

\subsection{Load Shedding Due to Insufficient Sources and Storage}

If the consumer grid is operating in islanding mode and on-site generators are also not generating enough to power up loads, then the main DC bus voltage $\left(V_{M B}\right)$ slope becomes negative, as shown in Figure $11 \mathrm{~b}$. The control model of PCmRC is designed in such a way that if the $V_{M B}$ reaches $355 \mathrm{~V}$ or less, each DCT connected to the main DC bus then goes into high-impedance by activating the DCT isolation switch as shown in Figure 2. If the DCT isolation switch is activated, then DCT switches off the non-critical load, as shown in Figure 11c,f and operates in the DCT isolation mode, as shown in Figure 11a. In DCT isolation mode, PCmRC utilizes the low voltage storage to ensure continuous supply for only critical loads without any interruption, as shown in Figure 11e. In Figure 11d, at $t=2.5 \mathrm{~s}$, as soon as $V_{M B}$ drops to $355 \mathrm{~V}, \mathrm{PCmRC}$ activates the low voltage storage at the same time 
to discharge on the sub LVDC side, which is shown with a negative sign in Figure 11d. Moreover, the sub LVDC bus voltage level rises proportionally to the storage voltage and will continue to supply power to the critical load until the storage ends or PCmRC starts operating again in the main grid interactive mode.

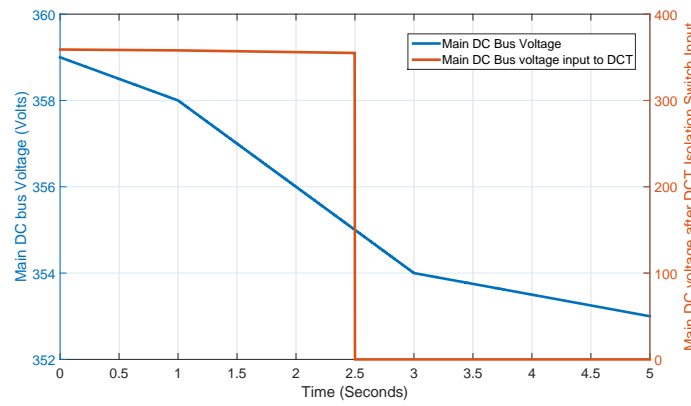

(a)

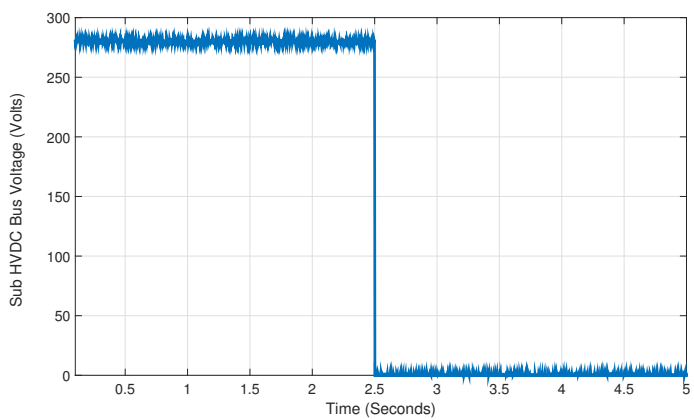

(c)

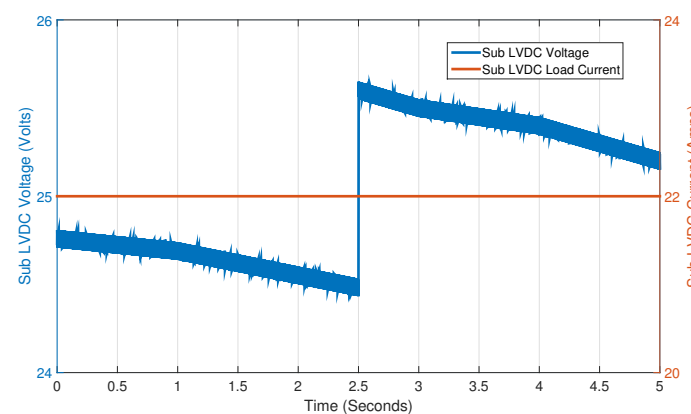

(e)

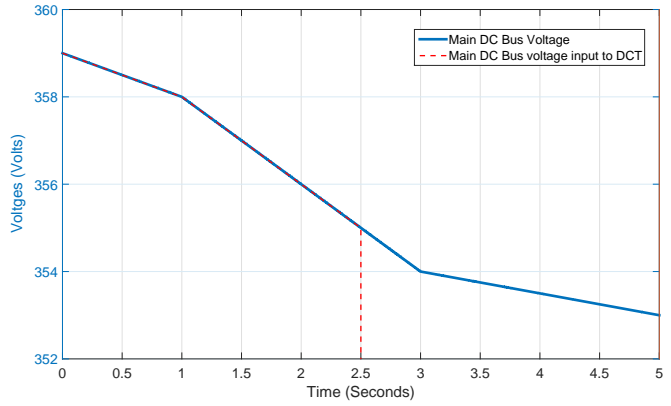

(b)

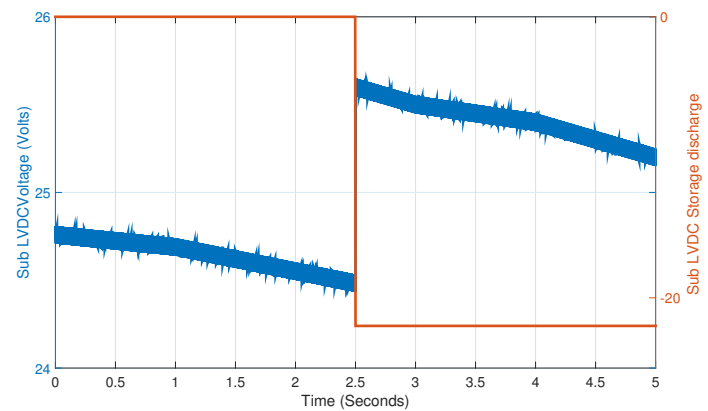

(d)

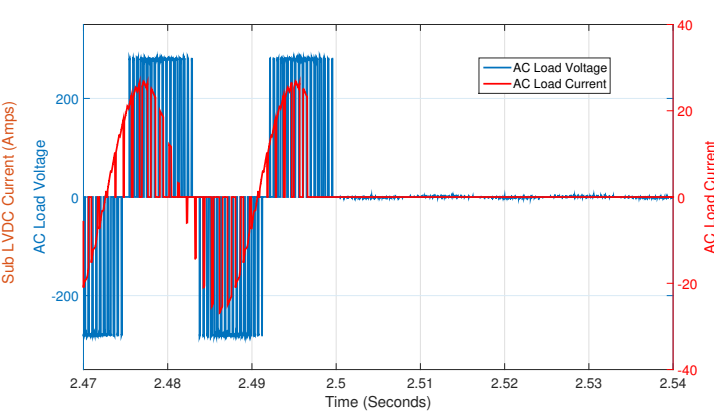

(f)

Figure 11. Simulation waveform of mode transition from the main grid interactive to the DCT isolated mode (a) main DC bus voltage before and after the DCT isolation switch; (b) main DC bus voltage; (c) sub HVDC voltage waveform; (d) sub LVDC bus with low voltage storage discharge; (e) sub LVDC bus voltage and current; and (f) AC load voltage and current.

\subsection{Load Shedding Due to Fault Occurring on the Demand Side}

In case, during normal grid operation, any fault occurs on the consumer side, the PCmRC immediately shuts off and isolates the respective DCT in order to ensure entire grid reliability. In Figure 12b, there is a high current spike generated on the sub LVDC port, and DCT immediately detects it, activates the DCT isolation switch, and whole consumer grid undergoes into the DCT isolation state within $2.5 \mathrm{~ms}$. However, in the DCT isolation state, the DCT shuts off all input and output ports and disconnects from the main DC bus, as shown in Figure 12a. Moreover, DCT 
switches-off the sub HVDC bus and inverter output at the same time to avoid any sort of damage on the consumer side, as shown in Figure 12c.

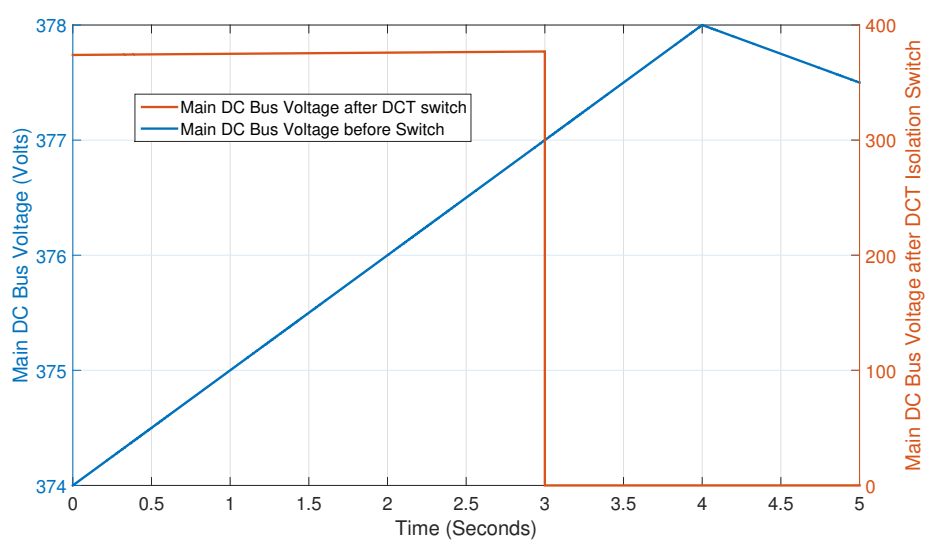

(a)

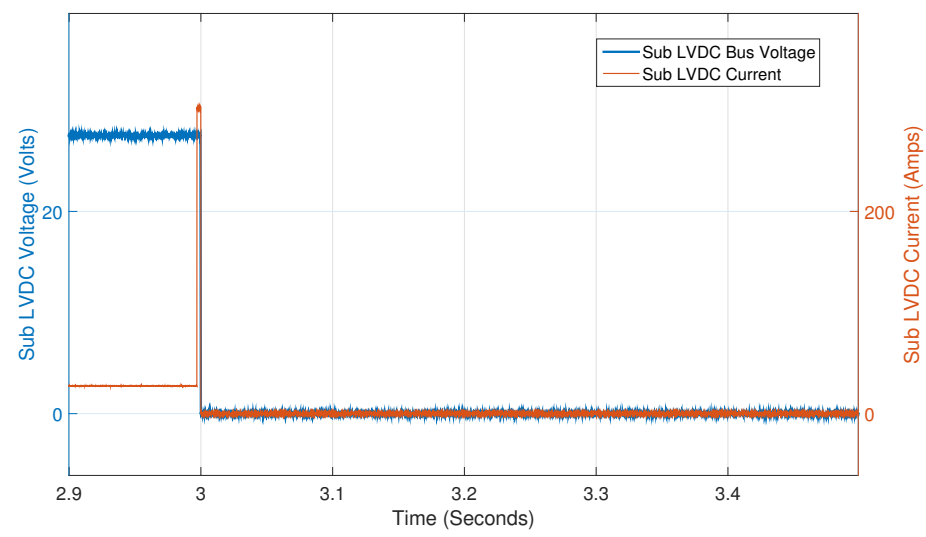

(b)

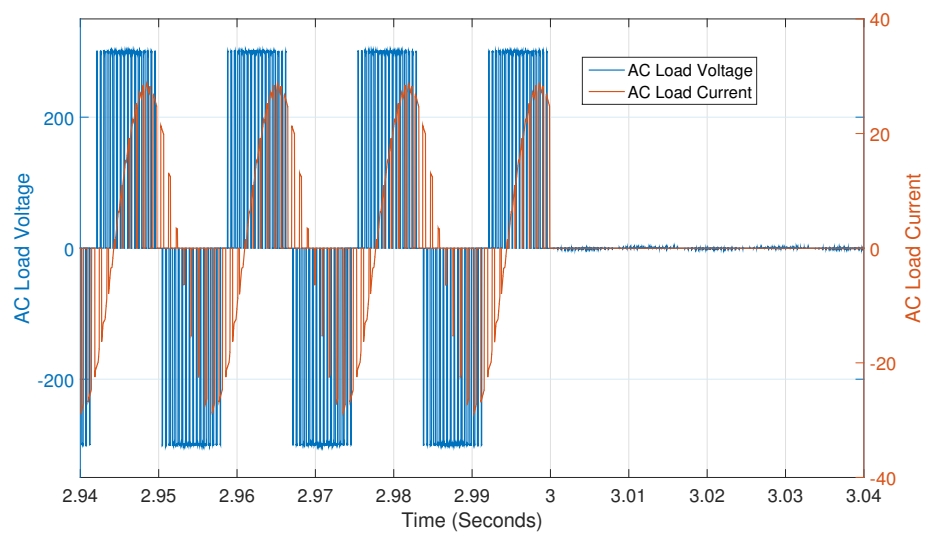

(c)

Figure 12. Simulation waveforms during fault condition on the consumer side (a) DC bus voltage before and after the DCT isolation switch; (b) high current spike on sub LVDC bus; and (c) AC load voltage and current before and after the fault.

\section{Conclusions}

In this paper, a unique power controlling, monitoring and routing strategy is presented, which is independent from the type of available renewable/distributed sources and storage. The proposed model provides seamless switching between operational modes without compromising grid reliability. 
Moreover, there would be no change in the control algorithm in the case of a DC grid operating in grid-connected, islanding, off-grid, or with or without on-site generators, which enables compatibility with the existing infrastructure and also handles any sort of situation flawlessly. A system model is constructed and key characteristics of the proposed model are examined by simulating various case studies, which validate the proposed concept of power management. However, as future work, the most important thing is to implement and deploy the scaled model DCT with two or more sources, at least one energy storage. and two loads both critical and non-critical. A practical test needs to be designed in order to evaluate the scaled DCT model in grid-connected and islanding modes with variable demand side power requirements. Proper investigation needs to be done related to overall stability when two or more DCT modules run parallel, in order to validate the active power sharing control design without affecting grid stability. In short, the DCT enabled PCmRC consumer grid model would be a promising key component in modern power distribution of consumer grids.

Author Contributions: Syed Ashad Mustufa Younus conceived the energy router for smart consumer grids and designed the experiments; Davide Brunelli supervised the entire work and the experiments performed; and Matteo Nardello and Pietro Tosato contributed to consolidate the work.

Conflicts of Interest: The authors declare no conflict of interest.

\section{References}

1. Jacobson, M.Z.; Delucchi, M.A. A path to sustainable energy by 2030. Sci. Am. 2009, 301, 58-65.

2. Piagi, P.; Lasseter, R. Autonomous control of microgrids. In Proceedings of the IEEE Power Engineering Society General Meeting, Tampa, FL, USA, 24-28 June 2006; p. 8.

3. Lasseter, R. MicroGrids. In Proceedings of the IEEE Power Engineering Society Winter Meeting, New York, NY, USA, 27-31 January 2002; pp. 305-308.

4. Porcarelli, D.; Brunelli, D.; Benini, L. Characterization of lithium-ion capacitors for low-power energy neutral wireless sensor networks. In Proceedings of the 2012 Ninth International Conference on Networked Sensing (INSS), Antwerp, Belgium, 11-14 June 2012; pp. 1-4.

5. Ciezki, J.; Ashton, R. Selection and stability issues associated with a navy shipboard DC zonal electric distribution system. IEEE Trans. Power Deliv. 2000, 15, 665-669.

6. Rossi, M.; Brunelli, D. Forecasting data centers power consumption with the Holt-Winters method. In Proceedings of the 2015 IEEE Workshop on Environmental, Energy, and Structural Monitoring Systems (EESMS), Trento, Italy, 9-10 July 2015; pp. 210-214.

7. Salomonsson, D.; Soder, L.; Sannino, A. An Adaptive Control System for a DC Microgrid for Data Centers. IEEE Trans. Ind. Appl. 2008, 44, 1910-1917.

8. Brunelli, D.; Tamburini, L. Residential load scheduling for energy cost minimization. In Proceedings of the 2014 IEEE International Energy Conference (ENERGYCON), Dubrovnik, Croatia, 13-16 May 2014; pp. 675-682.

9. Sechilariu, M.; Wang, B.; Locment, F. Building integrated photovoltaic system with energy storage and smart grid communication. IEEE Trans. Ind. Electron. 2013, 60, 1607-1618.

10. Dondi, D.; Brunelli, D.; Benini, L.; Pavan, P.; Bertacchini, A.; Larcher, L. Photovoltaic cell modeling for solar energy powered sensor networks. In Proceedings of the 2007 2nd International Workshop on Advances in Sensors and Interface, Bari, Italy, 26-27 June 2007; pp. 1-6.

11. Brunelli, D.; Rossi, M. Smart Grid Configuration Tool for HEES systems in smart city districts. In Proceedings of the 2016 International Symposium on Power Electronics, Electrical Drives, Automation and Motion (SPEEDAM), Anacapri, Italy, 22-24 June 2016; pp. 137-142.

12. Li, F.; Qiao, W.; Sun, H.; Wan, H.; Wang, J.; Xia, Y.; Xu, Z.; Zhang, P. Smart transmission grid: Vision and framework. IEEE Trans. Smart Grid 2010, 1, 168-177.

13. Engel, S.P.; Soltau, N.; Stagge, H.; Doncker, R.W.D. Dynamic and balanced control of three-phase high-power dual-active bridge DC 2013; DC converters in DC-Grid applications. IEEE Trans. Power Electron. 2013, 28, 1880-1889.

14. Xu, L.; Chen, D. Control and operation of a DC microgrid with variable generation and energy storage. IEEE Trans. Power Deliv. 2011, 26, 2513-2522. 
15. She, X.; Huang, A.Q.; Lukic, S.; Baran, M.E. On integration of solid-state transformer with zonal DC microgrid. IEEE Trans. Smart Grid 2012, 3, 975-985.

16. Jiang, W.; Fahimi, B. Active current sharing and source management in fuel cell-battery hybrid power system. IEEE Trans. Ind. Electron. 2010, 57, 752-761.

17. Planas, E.; Gil-de Muro, A.; Andreu, J.; Kortabarria, I.; Martínez de Alegría, I. General aspects, hierarchical controls and droop methods in microgrids: A review. Renew. Sustain. Energy Rev. 2013, 17, 147-159.

18. Schonberger, J.; Duke, R.; Round, S. DC-Bus signaling: A distributed control strategy for a hybrid renewable nanogrid. IEEE Trans. Ind. Electron. 2006, 53, 1453-1460.

19. Chen, D.; Xu, L.; Yao, L. DC voltage variation based autonomous control of DC microgrids. IEEE Trans. Power Del. 2013, 28, 637-648.

20. Wang, X.; Guerrero, J.M.; Blaabjerg, F.; Chen, Z. A review of power electronics based microgrids. J. Power Electron. 2012, 12, 181-192.

21. Rossi, M.; Toppano, A.; Brunelli, D. Real-time optimization of the battery banks lifetime in Hybrid Residential Electrical Systems. In Proceedings of the 2014 Design, Automation Test in Europe Conference and Exhibition (DATE), Dresden, Germany, 24-28 March 2014; pp. 1-6.

22. Ito, Y.; Zhongqing, Y.; Akagi, H. DC microgrid based distribution power generation system. In Proceedings of the 4th International Power Electronics and Motion Control Conference, Xi'an, China, 14-16 August 2004; pp. 1740-1745.

23. Rossi, M.; Brunelli, D. Electricity demand forecasting of single residential units. In Proceedings of the 2013 IEEE Workshop on Environmental Energy and Structural Monitoring Systems, Trento, Italy, 11-12 September 2013; pp. 1-6.

24. Yu, X.; She, X.; Zhou, X.; Huang, A. Power management for DC microgrid enabled by solid-state transformer. IEEE Trans. Smart Grid 2013, 5, 954-965.

25. Alliance, E. EMerge Alliance is a member of the U.S. Green Building Council, 2015. Available online: http:/ / www.informationweek.com/news/201202317 (accessed on 14 March 2015).

26. Systems Evaluation Group-Low Voltage Direct Current Applications, Distribution and Safety for Use in Developed and Developing Economies; International Electrotechnical Commission: Geneva, Switzerland, 2016.

27. She, X.; Huang, A.Q.; Wang, G. 3-D space modulation with voltage balancing capability for a cascaded seven-level converter in a solid-state transformer. IEEE Trans. Power Electron. 2011, 26, 3778-3789.

28. ICF International. Energy 2016 Buildings-European Commission: Energy Performance of Buildings Directive Compliance Study; ICF International: Fairfax, VA, USA, 2015.

29. Khorsandi, A.; Ashourloo, M.; Mokhtari, H. A decentralized control method for a low-voltage DC microgrid. IEEE Trans. Energy Convers. 2014, 29, 793-801.

30. Liu, X.; Wang, P.; Loh, P.C. A hybrid AC/DC microgrid and its coordination control. IEEE Trans. Smart Grid 2011, 2, 278-286.

31. Porcarelli, D.; Brunelli, D.; Benini, L. Clamp-and-Forget: A self-sustainable non-invasive wireless sensor node for smart metering applications. Microelectron. J. 2014, 45, 1671-1678.

(C) 2017 by the authors; licensee MDPI, Basel, Switzerland. This article is an open access article distributed under the terms and conditions of the Creative Commons Attribution (CC BY) license (http:/ / creativecommons.org/licenses/by/4.0/). 